Research Article

\title{
Prognostic Value of lncRNA PVT1 for Patients with Gastric Cancer: A Meta-Analysis
}

\author{
Jinyong Hao $\mathbb{D},{ }^{1,2}$ Bo Yuan, ${ }^{3}$ Yani Gou, ${ }^{1,2}$ Jichun Ma, ${ }^{4}$ and Xiaojun Huang $\mathbb{D}^{1,2}$ \\ ${ }^{1}$ Department of Gastroenterology, Lanzhou University Second Hospital, Lanzhou, China \\ ${ }^{2}$ Gansu Provincial Digestive Endoscopy Engineering Research Center, Lanzhou, China \\ ${ }^{3}$ Department of General Surgery, Lanzhou University Second Hospital, Lanzhou, China \\ ${ }^{4}$ First Clinical Medical College, Lanzhou University, Lanzhou, China \\ Correspondence should be addressed to Xiaojun Huang; huangxj@lzu.edu.cn
}

Received 2 March 2021; Revised 24 September 2021; Accepted 15 October 2021; Published 2 December 2021

Academic Editor: Muhammad Tahir ul Qamar

Copyright (c) 2021 Jinyong Hao et al. This is an open access article distributed under the Creative Commons Attribution License, which permits unrestricted use, distribution, and reproduction in any medium, provided the original work is properly cited.

Objective. To evaluate the prognostic value of lncRNA PVT1 for patients with gastric cancer. Methods. A comprehensive literature searching was performed in PubMed, Cochrane Library, Web of Science, Embase, CNKI, CBM, and Wanfang Database to identify published studies on the expression level of lncRNA PVT1 in human gastric cancer. STATA 12.0 was conducted to perform the meta-analysis. Clinical outcomes including patients' age, genders, TNM stage, OS, and DFS were assessed in the study. Results. A total of 8 studies involving 747 patients were included in this meta-analysis. The results of meta-analysis showed that higher expression level of lncRNA PVT1 was associated with GC patients' gender (for male: OR = 2.27, 95\% CI: 1.67 3.07, $P=0.000$ ), invasion depth (for T3 4: $\mathrm{OR}=3.98,95 \% \mathrm{CI}: 2.85 \sim 5.56, P=0.000$ ), poorer OS (HR=1.68, 95\% CI: $1.43 \sim 1.97, P=0.000$ ), and DFS (HR $=1.74,95 \%$ CI: 1.44 2.08, $P=0.000$ ). Conclusion. Higher expression level of lncRNA PVT1 is significantly associated with GC patients' gender, invasion depth, poorer OS, and worse DFS. lncRNA PVT1 might act as a novel predictive biomarker of poor prognosis and clinicopathological characteristics for gastric cancer.

\section{Introduction}

Long noncoding RNAs (lncRNAs) are a class of RNA molecules with a length of more than 200 nucleotides [1]. Due to the lack of open reading frames, they cannot encode proteins. In the early stage, they were considered as "junk sequences" in the process of gene transcription [2]. Although lncRNAs do not encode proteins, they can still regulate gene expression through a variety of mechanisms at the epigenetic, transcriptional, and posttranscriptional levels, which become one of the research hotspots in recent years [3]. A large number of studies have shown that lncRNAs play an important role in the invasion, migration, and metastasis of tumors $[4,5]$. Among them, plasma tumor variant translocation 1 (PVT1) was reported as a key biomarker of prognosis for patients with gastric cancer (GC) [6].

Numerous studies have reported that PVT1 showed higher expression in gastric cancer tissues and cell lines, can promote the proliferation and invasion of GC cells, and is closely related to advanced tumor stage and lymph node metastasis $[7,8]$. However, previous published studies have been limited by low study sample size; the association between clinical outcomes and PVT1 expression remains unclear. Thus, we conducted a meta-analysis with the aim to evaluate the prognostic value of PVT1 for patients with GC.

\section{Methods}

2.1. Search Strategy. A comprehensive literature searching was performed in PubMed, Cochrane Library, Web of Science, Embase, CNKI, CBM, and Wanfang Database to identify published studies on the expression level of lncRNA PVT1 in human gastric cancer in English and Chinese language. The following search terms were used in online databases: "gastric cancer", "stomach cancer", "gastric 


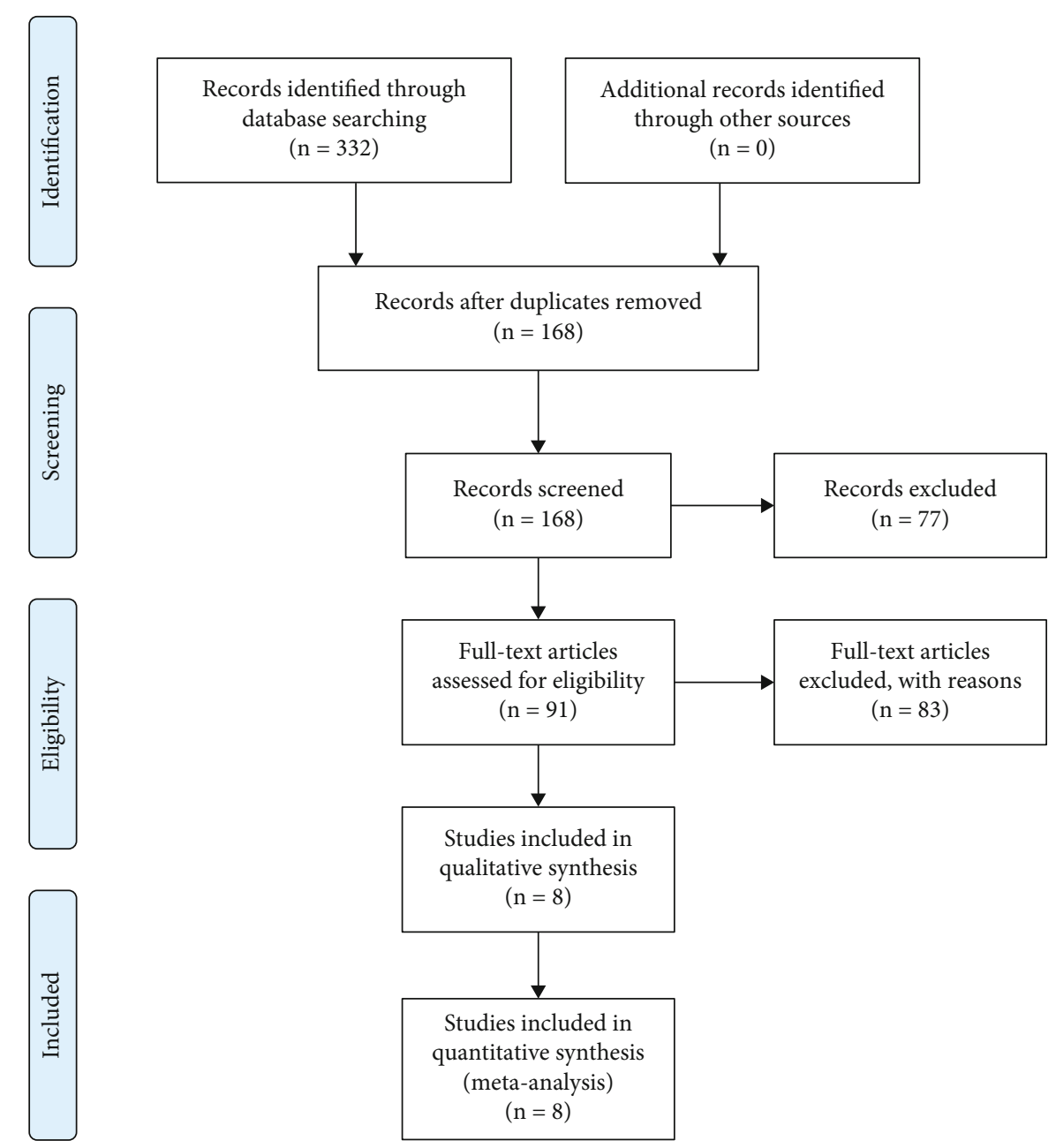

Figure 1: PRISMA flowchart of literature selection.

TABLE 1: The characteristics of included studies.

\begin{tabular}{|c|c|c|c|c|c|c|c|}
\hline Study & Country & Tumor stage & Sample size & Cut-off value & Follow-up (months, mean) & Detection methods & NOS score \\
\hline Ding 2014 & China & I-IV & 31 & $\mathrm{~T} / \mathrm{N}>1$ & NR & qRT-PCR & 7 \\
\hline Kong 2015 & China & I-IV & 80 & Mean & NR & qRT-PCR & 8 \\
\hline Yuan 2016 & China & I-IV & 111 & Mean & NR & qRT-PCR & 7 \\
\hline Xu 2017 & China & I-IV & 190 & Mean & 32.34 & qRT-PCR & 8 \\
\hline Huang 2017 & China & I-IV & 68 & Mean & NR & qRT-PCR & 8 \\
\hline Chen 2017 & China & I-IV & 187 & NR & 26.44 & qRT-PCR & 7 \\
\hline Niu 2020 & China & I-IV & 50 & NR & NR & qRT-PCR & 7 \\
\hline Wang 2020 & China & I-IV & 30 & NR & NR & qRT-PCR & 7 \\
\hline
\end{tabular}

NR: not reported; qRT-PCR: quantitative real-time PCR.

neoplasm", "long non-coding RNA", "lncRNA", "plasma tumor variant translocation 1", "PVT1", "clinical outcome", "prognosis", "overall survival", and "disease free survival". The literature search was undertaken from databases' construction to April 30, 2020. The meta-analysis was conducted according to the guideline of the Preferred Reporting Items for Systematic Reviews and Meta-Analysis (PRISMA).
2.2. Inclusion Criteria. Two reviewers screened and selected all search records on publication titles, abstracts, and full manuscript. Inclusion criteria were as follows: (1) all studies were published in English and Chinese, and full text is available on the online databases; (2) patients were diagnosed with gastric cancer in any TNM stage; (3) the expression level of lncRNA PVT1 was reported in studies, and lncRNA PVT1 was detected by established molecular methods, such 


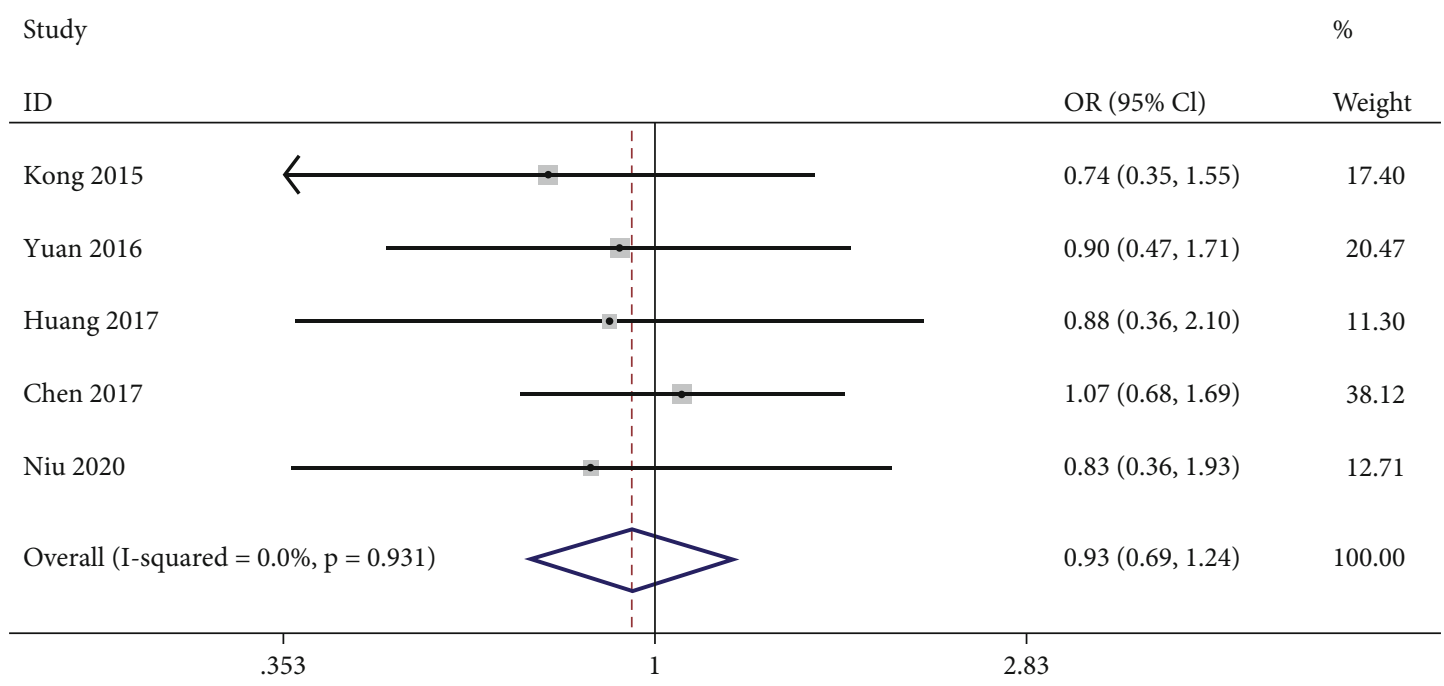

FIGURe 2: Meta-analysis of association between high expression level of lncRNA PVT1 and GC patients' age ( $>50$ yrs vs. $<50$ yrs).

\begin{tabular}{|c|c|c|}
\hline \multirow{2}{*}{$\begin{array}{l}\text { Study } \\
\text { ID }\end{array}$} & \multirow[b]{2}{*}{ OR $(95 \% \mathrm{Cl})$} & \multirow{2}{*}{$\begin{array}{l}\% \\
\text { Weight }\end{array}$} \\
\hline & & \\
\hline Ding 2014 & $1.38(0.45,4.18)$ & 9.30 \\
\hline Kong 2015 & $1.50(0.69,3.24)$ & 18.60 \\
\hline Yuan 2016 & $2.06(1.05,4.04)$ & 20.93 \\
\hline Huang 2017 & $1.14(0.48,2.75)$ & 16.28 \\
\hline Chen 2017 & $4.09(2.40,6.98)$ & 25.58 \\
\hline Niu 2020 & $2.13(0.78,5.82)$ & 9.30 \\
\hline Overall $(\mathrm{I}$-squared $=44.5 \%, \mathrm{p}=0.109)$ & $2.27(1.67,3.07)$ & 100.00 \\
\hline .143 & 98 & \\
\hline
\end{tabular}

Figure 3: Meta-analysis of association between high expression level of lncRNA PVT1 and GC patients' genders (male vs. female).

as qRT-PCR and western blot; (4) studies included in metaanalysis demonstrated the correlation between lncRNA PVT1 expression level and GC patients' clinical characteristics, survival data, and prognostic value; (5) sufficient statistical analysis was required, including overall survival (OS) and disease-free survival (DFS) and their hazard ratios (HRs) and 95\% confidence interval (95\% CI). KaplanMeier survival curves were also necessary.

2.3. Data Extraction. Two researchers extracted the required data from included studies, including (1) characteristics of included studies: study title, the year of publication, authors, country, tumor stage, sample size, cut-off value, follow-up duration, and lncRNA detection methods; (2) clinical outcomes: patients' age, genders, and TNM stage; and (3) survival data: OS, DFS, HR value, and 95\% CIs.
2.4. Quality Assessment. The quality of included studies was evaluated according to the Newcastle-Ottawa Scale (NOS) [9] by two reviewers independently. Any disagreements were discussed and resolved by another reviewer. NOS score ranged from 0 to 9; it was regarded as a high quality if one is with a NOS score $>7$.

2.5. Statistical Analysis. STATA 12.0 was conducted to perform the meta-analysis. Chi-based $Q$-test and $I^{2}$ test were used to assess the statistical heterogeneity between studies. When $I^{2}<50 \%$ or $Q$-test $P>0.1$, there is no heterogeneity in the data analysis; the fixed-effect model was adopted to perform the meta-analysis. Otherwise, the random-effect model was used in the meta-analysis. If the results are heterogeneous, subgroup analysis of possible factors that may lead to heterogeneity was undertaken. Sensitivity analysis 


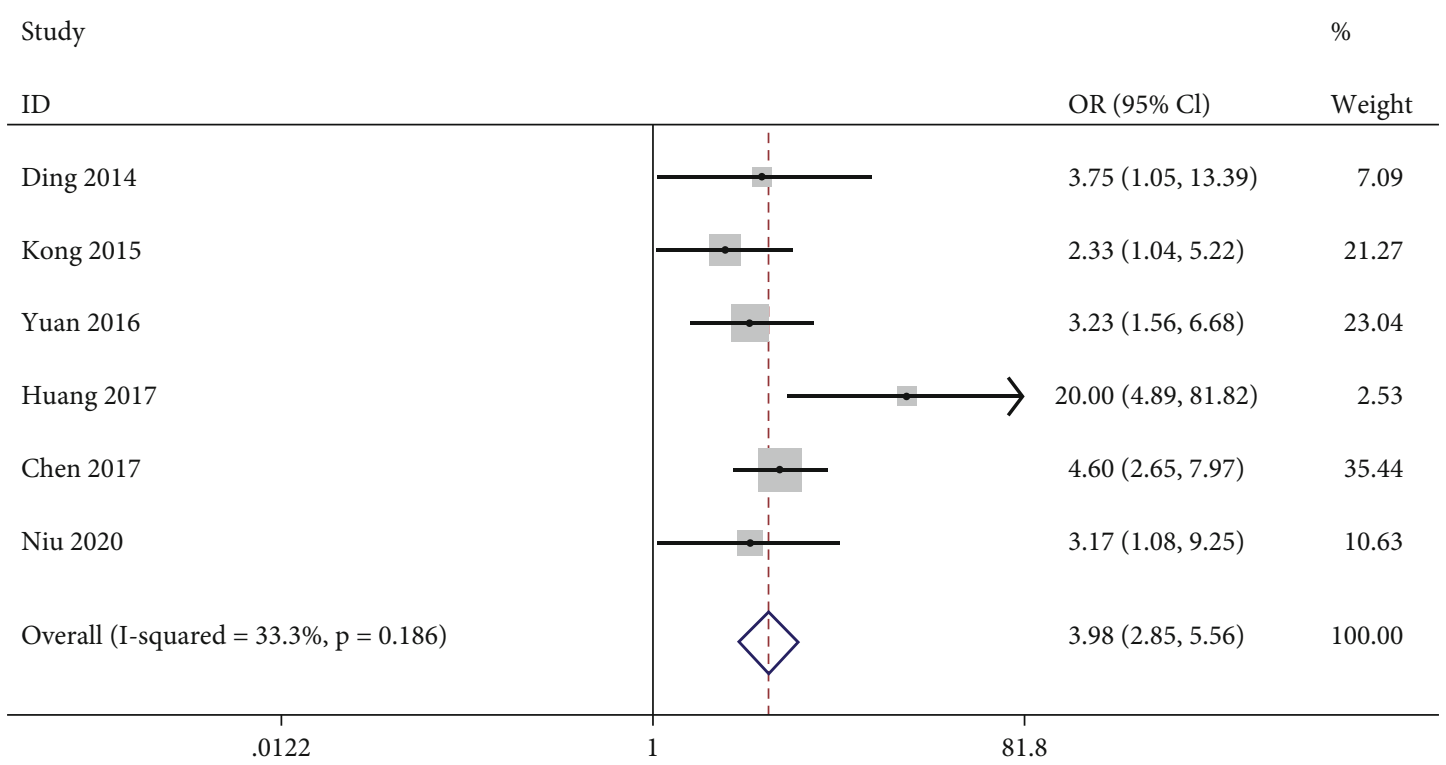

Figure 4: Meta-analysis of association between lncRNA PVT1 expression level and invasion depth (T3 4 vs. T1 2).

\begin{tabular}{|c|c|c|}
\hline Study & & $\%$ \\
\hline ID & $\mathrm{HR}(95 \% \mathrm{Cl})$ & Weight \\
\hline Kong 2015 & $2.09(1.08,4.08)$ & 5.79 \\
\hline Yuan 2016 & $2.27(1.06,4.88)$ & 4.38 \\
\hline Xu 2017 & $1.64(1.15,2.39)$ & 19.10 \\
\hline Chen 2017 & $1.49(1.01,2.21)$ & 16.67 \\
\hline Wang 2020 & $1.67(1.34,2.07)$ & 54.05 \\
\hline Overall $(\mathrm{I}$-squared $=0.0 \%, \mathrm{p}=0.846)$ & $1.68(1.43,1.97)$ & 100.00 \\
\hline
\end{tabular}

FIGURE 5: Meta-analysis of association between lncRNA PVT1 expression level and overall survival (high expression level vs. low expression level).

was also conducted to assess the ability of the combined results and to determine the source of any heterogeneity. And Begg's test was also used to evaluate the publication bias.

\section{Results}

3.1. The Characteristics of Included Studies. A systematic literature search was performed in databases. As shown in Figure 1, a total of 332 publications were identified from online databases. 164 duplicates were removed by Endnote $\mathrm{X} 17$, and 77 records were excluded after screening the titles and abstracts. 91 publications were dealt with reading the full text. Based on the inclusion criteria, 8 studies [10-17] were included in the present meta-analysis.
Of the eight studies on the association between expression level of lncRNA PVT1 and GC patients identified, 747 patients were included in this meta-analysis. All of the included studies are from China and reported the data on stage I-IV GC patients. Five of them reported the cut-off value, and the qRT-PCR method was used to detect the expression level of lncRNA PVT1 in all included publications. The results of quality assessment for included studies ranged from 7 to 8 , which demonstrated a high quality for the 8 included publications (Table 1).

3.2. Association between IncRNA PVT1 Expression Level and GC Patients' Age. Five of the included studies reported the association between lncRNA PVT1 expression level and GC patients' age. No heterogeneity between studies was observed $\left(I^{2}=0.0 \%, P=0.931\right)$. Meta-analysis in a fixed- 


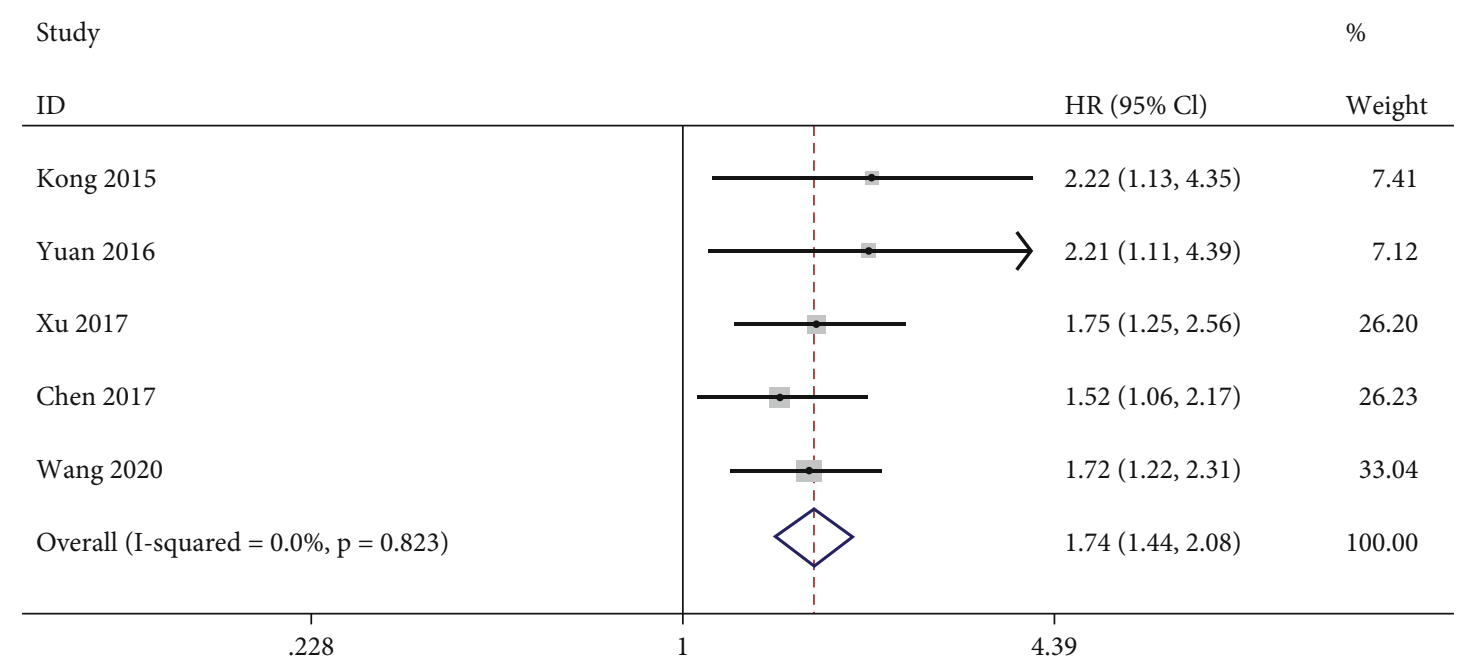

FIGURE 6: Meta-analysis of association between lncRNA PVT1 expression level and DFS (high expression level vs. low expression level).

effect model showed that there is no relationship between high expression level of lncRNA PVT1 and GC patients' age $(\mathrm{OR}=0.93,95 \%$ CI: 0.68 1.24, $P=0.606)$ (Figure 2).

3.3. Association between IncRNA PVT1 Expression Level and GC Genders. Six studies reported the association between lncRNA PVT1 expression level and GC genders. There is no heterogeneity between studies $\left(I^{2}=44.5 \%, P=0.109\right)$; a fixed-effect model was conducted to perform meta-analysis. The results showed that high expression level of lncRNA PVT1 was associated with male gender $(\mathrm{OR}=2.27,95 \%$ CI: 1.67 3.07, $P=0.000$ ) (Figure 3).

3.4. Association between IncRNA PVT1 Expression Level and Invasion Depth (T3 4 vs. T1 2). Six of the included studies reported association between lncRNA PVT1 expression level and invasion depth. Meta-analysis in a fixed-effect model (study heterogeneity: $I^{2}=33.3 \%, P=0.186$ ) showed that high expression level of lncRNA PVT1 was associated with T3 4 (OR $=3.98,95 \%$ CI: 2.85 5.56, $P=0.000)$ (Figure 4).

3.5. Association between IncRNA PVT1 Expression Level and Overall Survival. Five of the included studies reported association between lncRNA PVT1 expression level and overall survival. Meta-analysis in a fixed-effect model $\left(I^{2}=0.0 \%, P\right.$ $=0.845$ ) showed that higher expression level of lncRNA PVT1 is associated with poorer OS for patients with GC $(\mathrm{HR}=1.68,95 \% \mathrm{CI}: 1.43 \sim 1.97, P=0.000)$ (Figure 5).

3.6. Association between IncRNA PVT1 Expression Level and Disease-Free Survival. Five of the included studies reported the association between lncRNA PVT1 expression level and DFS. Meta-analysis performed in a fixed-effect model $\left(I^{2}=0.0 \%, P=0.823\right)$ showed that high expression level of IncRNA PVT1 was associated with poorer DFS for GC patients $\quad(\mathrm{HR}=1.74, \quad 95 \% \quad \mathrm{CI}: \quad 1.44 \sim 2.08, \quad P=0.000)$ (Figure 6).

\section{Discussion}

The length of lncRNA PVT1 is about $1716 \mathrm{NT}$, which is located in chromosome 8q24 region and sense strand [18]. It spans more than $300 \mathrm{~KB}$ genome interval [19]. In tumor cells, chromosome 8q24 region is the highest target of DNA copy number amplification, and its abnormal amplification often indicates high risk of cancer [20]. The abnormal expression of lncRNA PVT1 is associated with many human tumors, such as lung cancer, gastric cancer, colorectal cancer, breast cancer, pancreatic cancer, and prostate cancer [21-24]. Different expressions of lncRNA PVT1 in various tumors indicate that it plays an important role in the occurrence and development of tumors.

Gastric cancer is one of the most common gastrointestinal tumors, which is the third leading cause of cancer-related death and accounting for $9 \%$ of the global cancer mortality [25]. In 2015, the number of confirmed cases of gastric cancer in China was about 679000, only second to lung cancer (733000), higher than esophageal cancer (478000) and liver cancer (466000) [26]. Although great progress has been made in the surgical treatment and chemotherapy of gastric cancer in recent years, due to the lack of specific symptoms and signs in the early stage of gastric cancer, most patients with gastric cancer are in the advanced stage at the time of diagnosis, which seriously affects the prognosis of patients [27]. The 5-year survival rate of patients with advanced gastric cancer is less than $10 \%$ [28]. Therefore, it is urgent to find a new biomarker for the diagnosis and prognosis of gastric cancer.

In present studies, the results of meta-analysis showed that higher expression level of lncRNA PVT1 was associated with GC patients' gender (for male: $\mathrm{OR}=2.27,95 \% \mathrm{CI}$ : 1.67 3.07, $P=0.000$ ), invasion depth (for T3 4: $\mathrm{OR}=3.98$, 95\% CI: $2.85 \sim 5.56, P=0.000)$, poorer OS $(\mathrm{HR}=1.68,95 \%$ CI: $1.43 \sim 1.97, P=0.000)$, and DFS (HR $=1.74,95 \% \mathrm{CI}$ : 1.44 2.08, $P=0.000$ ). Previous meta-analysis [29] also reported that cancer patients with high PVT1 expression had a poorer overall survival $(\mathrm{HR}=2.07,95 \% \mathrm{CI}$ : 1.40 - 
2.74, $P=0.000)$ and a worse disease-free survival $(\mathrm{HR}=2.10$ , 95\% CI: $0.96-3.23, P=0.000)$. Therefore, by detecting the expression of IncRNA PVT1 in tumor tissue, we can have a comprehensive analysis and evaluation of the patient's condition and prognosis and provide a reference for the realization of individualized treatment.

Some limitations in our meta-analysis also should be taken into account. Firstly, the cut-off value for distinguishing low and high lncRNA PVT1 expression reported in included studies was different. Secondly, we searched databases in English and Chinese so that some relevant publications could have been ignored. Thirdly, studies included in our meta-analysis are from China; more other multicenter clinical studies from other countries should be conducted to confirm these results.

In conclusion, higher expression level of lncRNA PVT1 is significantly associated with GC patients' gender, invasion depth, poorer OS, and worse DFS. IncRNA PVT1 might act as a novel predictive biomarker of poor prognosis and clinicopathological characteristics for gastric cancer.

\section{Data Availability}

All data and analytic methods are available by e-mail request to the corresponding authors when required.

\section{Ethical Approval}

Ethical approval is not required as a literature-based study in this meta-analysis.

\section{Conflicts of Interest}

The authors have no conflicts of interest to disclose.

\section{Authors' Contributions}

Jinyong Hao and Bo Yuan contributed equally to this work.

\section{Acknowledgments}

The study was supported by Key Research and Development Project of Gansu Province (No. 20YF8FA076) and Youth Science and Technology Plan Project of Gansu Province (No. 20JR10RA755).

\section{References}

[1] X. Y. Fang, H. F. Pan, R. X. Leng, and D. Q. Ye, "Long noncoding RNAs: Novel insights into gastric cancer," Cancer Letters, vol. 356, no. 2, pp. 357-366, 2015.

[2] R. Miranda-Castro, "Long noncoding RNAs: from genomic junk to rising stars in the early detection of cancer," Analytical and Bioanalytical Chemistry, vol. 411, no. 19, pp. 4265-4275, 2019.

[3] B. Sun, C. Liu, H. Li et al., "Research progress on the interactions between long non-coding RNAs and microRNAs in human cancer (review)," Oncology Letters, vol. 19, no. 1, p. 132, 2019.
[4] M. Sarfi, M. Abbastabar, and E. Khalili, "Long noncoding RNAs biomarker-based cancer assessment," Journal of Cellular Physiology, vol. 234, no. 10, pp. 16971-16986, 2019.

[5] J. Li, R. Liu, S. Tang et al., "The effect of long noncoding RNAs HOX transcript antisense intergenic RNA single-nucleotide polymorphisms on breast cancer, cervical cancer, and ovarian cancer susceptibility: a meta-analysis," Journal of Cellular Biochemistry, vol. 24, no. 6, p. 212, 2019.

[6] S. Jones, "Transcriptional profiling identifies the long noncoding RNA plasmacytoma variant translocation (PVT1) as a novel regulator of the asthmatic phenotype in human airway smooth muscle," The Journal of Allergy and Clinical Immunology, vol. 139, no. 3, pp. 780-789, 2017.

[7] Y. R. Yang, S. Z. Zang, C. L. Zhong, Y. X. Li, S. S. Zhao, and $\mathrm{X}$. J. Feng, "Increased expression of the lncRNA PVT1 promotes tumorigenesis in non-small cell lung cancer," International Journal of Clinical \& Experimental Pathology, vol. 7, no. 10, p. 6929, 2014.

[8] F. Wang, J.-H. Yuan, S.-B. Wang et al., "Oncofetal long noncoding RNA PVT1 promotes proliferation and stem cell-like property of hepatocellular carcinoma cells by stabilizing NOP2," Hepatology, vol. 60, no. 4, pp. 1278-1290, 2014.

[9] K. L. Lo, D. Mertz, and M. Loeb, "Newcastle-Ottawa Scale: comparing reviewers' to authors' assessments," BMC Medical Research Methodology, vol. 14, no. 1, p. 45, 2014.

[10] J. Chen, Y. Li, Q. Zheng et al., "Circular RNA profile identifies circPVT1 as a proliferative factor and prognostic marker in gastric cancer," Cancer Letters, vol. 388, pp. 208-219, 2017.

[11] J. Ding, D. Li, M. Gong et al., "Expression and clinical significance of the long non-coding RNA PVT1 in human gastric cancer," Oncotargets and Therapy, vol. 2014, pp. 1625-1630, 2014.

[12] T. Huang, H. W. Liu, J. Q. Chen et al., "The long noncoding RNA PVT1 functions as a competing endogenous RNA by sponging miR-186 in gastric cancer," Biomedicine \& Pharmacotherapy, vol. 88, pp. 302-308, 2017.

[13] R. Kong, E. B. Zhang, D. D. Yin et al., "Long noncoding RNA PVT1 indicates a poor prognosis of gastric cancer and promotes cell proliferation through epigenetically regulating p15 and p16," Molecular Cancer, vol. 14, no. 1, p. 82, 2015.

[14] J. Niu, X. Song, and X. Zhang, "Regulation of lncRNA PVT1 on miR-125 in metastasis of gastric cancer cells," Oncology Letters, vol. 19, no. 2, pp. 1261-1266, 2020.

[15] L. Wang, B. Xiao, T. Yu et al., "IncRNA PVT1 promotes the migration of gastric cancer by functioning as ceRNA of miR$30 \mathrm{a}$ and regulating Snail," Journal of Cellular Physiology, vol. 236, pp. 536-548, 2020.

[16] M. D. Xu, Y. Wang, W. Weng et al., "A positive feedback loop of lncRNA-PVT1 and FOXM1 facilitates gastric cancer growth and invasion," Clinical Cancer Research: an official journal of the American Association for Cancer Research, vol. 23, no. 8, pp. 2071-2080, 2017.

[17] C. L. Yuan, H. Li, L. Zhu, Z. Liu, J. Zhou, and Y. Shu, “Aberrant expression of long noncoding RNA PVT1 and its diagnostic and prognostic significance in patients with gastric cancer," Neoplasma, vol. 63, no. 3, pp. 442-449, 2016.

[18] C. Huang, W. Yu, Q. Wang et al., "Increased expression of the lncRNA PVT1 is associated with poor prognosis in pancreatic cancer patients," Minerva Medica, vol. 106, no. 3, p. 143, 2015.

[19] C. J. Shen, Y. M. Cheng, and C. L. Wang, "LncRNA PVT1 epigenetically silences miR-195 and modulates EMT and 
chemoresistance in cervical cancer cells," Journal of Drug Targeting, vol. 25, no. 7, pp. 637-644, 2017.

[20] F. Wang, X. Ji, J. Wang et al., "LncRNA PVT1 enhances proliferation and cisplatin resistance via regulating miR-194-5p/ HIF1a axis in oral squamous cell carcinoma," Oncotargets and Therapy, vol. Volume 13, pp. 243-252, 2020.

[21] K. Yi, M. Hou, J. Yuan et al., "LncRNA PVT1 epigenetically stabilizes and post-transcriptionally regulates FOXM1 by acting as a microRNA sponge and thus promotes malignant behaviors of ovarian cancer cells," American Journal of Translational Research, vol. 12, no. 6, p. 76, 2020.

[22] M. Iden, S. Fye, K. Li, T. Chowdhury, R. Ramchandran, and J. S. Rader, "The IncRNA PVT1 contributes to the cervical cancer phenotype and associates with poor patient prognosis," PLoS One, vol. 11, no. 5, article e0156274, 2016.

[23] C. Huang, S. Liu, H. Wang, Z. Zhang, Q. Yang, and F. Gao, "LncRNA PVT1 overexpression is a poor prognostic biomarker and regulates migration and invasion in small cell lung cancer," American Journal of Translational Research, vol. 8, no. 11, pp. 5025-5034, 2016.

[24] K. Yoshida, S. Toden, P. Ravindranathan, H. Han, and A. Goel, "Curcumin sensitizes pancreatic cancer cells to gemcitabine by attenuating PRC2 subunit EZH2, and the lncRNA PVT1 expression," Carcinogenesis, vol. 38, no. 10, pp. 1036-1046, 2017.

[25] F. Kopp and J. T. Mendell, "Functional Classification and Experimental Dissection of Long Noncoding RNAs," Cell, vol. 172, no. 3, pp. 393-407, 2018.

[26] G. Spolverato and T. M. Pawlik, "Clinicopathological evaluation of recurrence in early gastric cancer," American Journal of Surgery, vol. 157, no. 3, pp. 202-207, 2019.

[27] I. B. Shin, S. J. Oh, and B. J. Suh, "Surgical outcomes and prognostic factors of gastric cancer surgery in octogenarians," Korean Journal of Clinical Oncology, vol. 15, no. 2, pp. 112120, 2019.

[28] K. K. Sun, Q. H. Wang, and Y. Y. Wu, "Challenges surrounding postoperative adjuvant chemotherapy for T2N0 gastric cancer (review)," Oncology Letters, vol. 20, no. 5, pp. 1-1, 2020.

[29] F. T. Liu, Q. Z. Xue, Z. M. Zhu et al., "Long noncoding RNA PVT1, a novel promising biomarker to predict lymph node metastasis and prognosis: a meta-analysis," Panminerva Medica, vol. 58, no. $2,2016$. 